\title{
Dilatonic dyon black hole solutions
}

\author{
M.E. Abishev ${ }^{1,2}$, K.A. Boshkayev ${ }^{1,2}$, V.D. Dzhunushaliev ${ }^{1,2}$ \\ and V.D. Ivashchuk ${ }^{3,4}$ \\ ${ }^{1}$ IETP, Al-Farabi KazNU, Almaty, 050040, Kazakhstan \\ ${ }^{2}$ Physical and Technical Faculty, Al-Farabi Kazakh National University, \\ al'-Farabi street, 71, Almaty 050040, Kazakhstan \\ ${ }^{3}$ Center for Gravitation and Fundamental Metrology, VNIIMS, \\ Ozyornaya St., 46, Moscow 119361, Russia, \\ ${ }^{4}$ Institute of Gravitation and Cosmology, \\ Peoples' Friendship University of Russia, \\ Miklukho-Maklaya St.,6, Moscow 117198, Russia
}

September 21, 2018

\begin{abstract}
Dilatonic black hole dyon solutions with arbitrary dilatonic coupling constant $\lambda \neq 0$ and canonical sign $\varepsilon=+1$ for scalar field kynetic term are considered. These solutions are defined up to solutions of two master equations for moduli funtions. For $\lambda^{2} \neq 1 / 2$ the solutions are extended to $\varepsilon= \pm 1$, where $\varepsilon=-1$ corresponds to ghost (phantom) scalar field. Some physical parameters of the solutions: gravitational mass, scalar charge, Hawking temperature, black hole area entropy and parametrized post-Newtonian (PPN) parameters $\beta$ and $\gamma$ are obtained. It is shown that PPN parameters do not depend on scalar field coupling $\lambda$ and $\varepsilon$. Two group of bounds on gravitational mass and scalar charge (for fixed and arbitrary extremality parameter $\mu>0$ ) are found by using a certain conjecture on parameters of solutions when $1+2 \lambda^{2} \varepsilon>0$. These bounds are verified numerically for certain examples. By product we are led to well-known lower bound on mass which was obtained earlier by Gibbons, Kastor, London, Townsend and Traschen by using spinor techniques.
\end{abstract}




\section{Introduction}

At present there exists an interest to spherically-symmetric solutions, e.g. black hole and black brane ones, related to Lie algebras and Toda chains, see [1]-[23] and references therein. These solutions appear in gravitational models with scalar fields and antisymmetric forms. Meanwhile special subclasses of low-dimensional (e.g. 4-dimensional) solutions were not considered in detail.

Here we consider a subclass of 4-dimensional dilatonic black hole solutions with electric and magnetic charges. We extend dilatonic black hole dyon solution from [18] to more general case, when dilatonic scalar field may be a ghost (or phantom) one. The ghost field appears in the action with a kinetic term of the "wrong sign". This implies the violation of the null energy condition $p+\rho \geq 0$. At the quantum level, such fields could form a "ghost condensate", which would be responsible for modified gravity laws in the infra-red limit [24]. Present observational data do not exclude this possiblity, and moreover under certain conditions the phantom scenario has a preference [25].

The main goal of our paper is the search of relations on physical parameters of dyonic black holes, e.g. bounds on gravitational mass $M$ and scalar charge $Q_{\varphi}$. This problem is solved here up to a conjecture, which states one to one (smooth) correspondence between the pair $\left(Q_{1}^{2}, Q_{2}^{2}\right)$, where $Q_{1}$ is electric charge and $Q_{2}$ is magnetic charge, and the pair $\left(P_{1}, P_{2}\right)$, where $P_{1}>0$ and $P_{2}>0$ are parameters of the solutions. This conjecture is believed to be valid for all $\lambda \neq 0$ in the case of ordinary scalar field and for $0<\lambda^{2}<1 / 2$ for the case of phantom scalar field. Here we verify the bounds by using numerical calculations.

\section{Black hole dyon solutions}

\subsection{Dyonic solutions}

We consider a model governed by the action

$$
S=\frac{1}{16 \pi G} \int d^{4} x \sqrt{|g|}\left\{R[g]-\varepsilon g^{\mu \nu} \partial_{\mu} \varphi \partial_{\nu} \varphi-\frac{1}{2} \exp (2 \lambda \varphi) F_{\mu \nu} F^{\mu \nu}\right\},
$$

where $g=g_{\mu \nu}(x) d x^{\mu} \otimes d x^{\nu}$ is metric, $\varphi$ is scalar field, $F=d A=\frac{1}{2} F_{\mu \nu} d x^{\mu} \wedge d x^{\nu}$ is Maxwell 2-form, $A=A_{\mu} d x^{\mu}, \varepsilon= \pm 1, G$ is gravitational constant, $\lambda \neq 0$ is a coupling constant and $|g|=\left|\operatorname{det}\left(g_{\mu \nu}\right)\right|$. Here we put $\lambda^{2} \neq 1 / 2$ for $\varepsilon=-1$. 
Let us consider a family of dyonic black hole solutions to the field equations corresponding to the action (2.1). These solutions are defined on the manifold

$$
M=(2 \mu,+\infty) \times S^{2} \times \mathbb{R},
$$

and have the following form

$$
\begin{aligned}
& d s^{2}=g_{\mu \nu} d x^{\mu} d x^{\nu}=\left(H_{1} H_{2}\right)^{h}\left\{-\left(H_{1} H_{2}\right)^{-2 h}\left(1-\frac{2 \mu}{R}\right) d t^{2}\right. \\
& \left.\quad+\frac{d R^{2}}{1-\frac{2 \mu}{R}}+R^{2} d \Omega_{2}^{2}\right\} \\
& \exp (\varphi)=\left(\frac{H_{1}}{H_{2}}\right)^{h \lambda \varepsilon}, \\
& F=\frac{Q_{1}}{R^{2}} H_{1}^{-2} H_{2}^{-a} d t \wedge d R+Q_{2} \tau .
\end{aligned}
$$

Here $Q_{1}$ and $Q_{2}$ are charges - electric and magnetic, respectively, $\mu>0$ is extremality parameter, $d \Omega_{2}^{2}=d \theta^{2}+\sin ^{2} \theta d \phi^{2}$ is canonical metric on the unit sphere $S^{2}(0<\theta<\pi, 0<\phi<2 \pi), \tau=\sin \theta d \theta \wedge d \phi$ is standard volume form on $S^{2}$,

$$
h=\frac{2}{1+2 \lambda^{2} \varepsilon},
$$

and

$$
a=2 \frac{1-2 \lambda^{2} \varepsilon}{1+2 \lambda^{2} \varepsilon} .
$$

Functions $H_{s}>0$ obey the equations

$$
R^{2} \frac{d}{d R}\left(R^{2} \frac{\left(1-\frac{2 \mu}{R}\right)}{H_{s}} \frac{d H_{s}}{d R}\right)=-h^{-1} Q_{s}^{2} \prod_{l=1,2} H_{l}^{-A_{s l}},
$$

with the following boundary conditions imposed

$$
H_{s} \rightarrow H_{s 0}>0,
$$

for $R \rightarrow 2 \mu$, and

$$
H_{s} \rightarrow 1 \text {, }
$$

for $R \rightarrow+\infty, s=1,2$. 
In 2.7 we denote

$$
\left(A_{s s^{\prime}}\right)=\left(\begin{array}{ll}
2 & a \\
a & 2
\end{array}\right)
$$

For the case $\varepsilon=1$ these solutions were presented earlier in [18. They may be obtained by using general formulae for non-extremal (intersecting) black brane solutions from [14, 15, 16, 17].

First boundary condition (2.8) guarantees (up to a possible additional demand on analicity of $H_{s}(R)$ in the vicinity of $R=2 \mu$ ) the existence of (regular) horizon at $R=2 \mu$ for the metric (2.2). Second condition (2.9) ensures an asymptotical (for $R \rightarrow+\infty$ ) flatness of the metric.

Equations (2.7) may be rewritten in the following form

$$
\frac{d}{d z}\left[(1-z) \frac{d y_{s}}{d z}\right]=-b q_{s}^{2} \exp \left(-2 y_{s}-a y_{\bar{s}}\right)
$$

$s=1,2$. Here and in what follows we use the following notations: $y_{s}=\ln H_{s}$, $z=2 \mu / R, q_{s}=Q_{s} /(2 \mu), b=h^{-1}$ and $\bar{s}=2,1$ for $s=1,2$, respectively. We are seeking solutions to equations 2.11 for $z \in(0,1)$ obeying

$$
\begin{gathered}
y_{s}(0)=0, \\
y_{s}(1)=y_{s 0},
\end{gathered}
$$

where $y_{s 0}=\ln H_{s 0}$ are finite (real) numbers, $s=1,2$. Here $z=0$ (or, more precisely $z=+0$ ) corresponds to infinity $(R=+\infty$ ), while $z=1$ (or, more rigorously, $z=1-0)$ corresponds to the horizon $(R=2 \mu)$.

Equations (2.11) with the finitness conditions on the horizon 2.13 imposed imply the following integral of motion:

$$
\begin{aligned}
(1-z)[ & {\left[\left(\frac{d y_{1}}{d z}\right)^{2}+\left(\frac{d y_{2}}{d z}\right)^{2}+a \frac{d y_{1}}{d z} \frac{d y_{2}}{d z}\right]+\frac{d y_{1}}{d z}+\frac{d y_{2}}{d z} } \\
& -b q_{1}^{2} \exp \left(-2 y_{1}-a y_{2}\right)-b q_{2}^{2} \exp \left(-2 y_{2}-a y_{1}\right)=0
\end{aligned}
$$

Equations (2.11) and (2.13) appear for special solutions to Toda-type equations [15, 16, 17]

$$
\frac{d^{2} z_{s}}{d u^{2}}=b Q_{s}^{2} \exp \left(2 z_{s}+a z_{\bar{s}}\right)
$$

for functions $z_{s}(u)=-y_{s}-\mu b u, s=1,2$, depending on harmonic radial variable $u$ : $\exp (-2 \mu u)=1-z$, with the following asymptotical behaviour 
for $u \rightarrow+\infty$ (on the horizon) imposed:

$$
z_{s}(u)=-\mu b u+z_{s 0}+o(1)
$$

where $z_{s 0}$ are constants, $s=1,2$. The energy integral of motion for (2.15), which is compatible with the asymptotic conditions (2.16),

$$
\begin{aligned}
E=\frac{h}{2}[ & \left.\left(\frac{d z_{1}}{d u}\right)^{2}+\left(\frac{d z_{2}}{d u}\right)^{2}+a \frac{d z_{1}}{d u} \frac{d z_{2}}{d u}\right] \\
& -\sum_{s=1,2} \frac{1}{2} Q_{s}^{2} \exp \left(2 z_{s}+a z_{\bar{s}}\right)=\mu^{2}
\end{aligned}
$$

leads us to relation 2.14.

Remark 1. Here we exclude the case $\lambda^{2}=1 / 2$ for $\varepsilon=-1$ from our consideration, since we deal with the finite value of the parameter $h$. But nevertheless, one can also obtain a sensible solution with a horizon for this peculiar case. This may be achieved by using another choice of moduli functions: $\bar{H}_{s}=H_{s}^{h}$ instead of $H_{s}$. The (implicit) solutions given by (2.2), (2.3), (2.4) and the master equations (2.7), rewritten in terms of new moduli functions $\bar{H}_{s}$, have a sensible limit for $\lambda^{2}=1 / 2$ and $\varepsilon=-1$. This special case may be a subject of a separate publication.

\section{Some integrable cases}

At present it seems impossible to find explicit solutions to the equations (2.7), (2.8), (2.9) analytically. One may try to seek the solutions in the form

$$
H_{s}=1+\sum_{k=1}^{\infty} P_{s}^{(k)}\left(\frac{1}{R}\right)^{k},
$$

where $P_{s}^{(k)}$ are constants, $k=1,2, \ldots$ and $s=1,2$.

Remark 2. The $1 / R$ expansion is widely used in gravitational physics sometimes without any indication whether the series like (3.1) is i) convergent, or ii) asymptotical one. Here the first possibility i) follows from analytical behaviour of functions $H_{s}$ with respect to $z=2 \mu / R$ in the vicinity of the point $z=0$, which is based on equations (2.14) and the non-degeneracy condition for the function $f(z)=1-z$ at $z=0$, i.e. $f(0)=1 \neq 0$. In 
what follows any of two assumptions: i) or ii) (or even more modest one: $H_{s}=1+P_{s} / R+o(1 / R)$, for $\left.R \rightarrow+\infty\right)$ is enough for our analysis.

Meanwhile, there exist at least two integrable configurations related to Lie algebras $A_{1}+A_{1}$ and $A_{2}$.

\section{$3.1\left(A_{1}+A_{1}\right)$-case}

Let

$$
\lambda^{2}=\frac{1}{2}, \quad \varepsilon=1
$$

This value of dilatonic coupling corresponds to string induced model. We get $h=1, a=0$ and hence 2.10 is the Cartan matrix for the Lie algebra $A_{1}+A_{1}\left(A_{1}=s l(2)\right)$. In this case

$$
H_{s}=1+\frac{P_{s}}{R}
$$

where

$$
P_{s}\left(P_{s}+2 \mu\right)=Q_{s}^{2}
$$

$s=1,2$. For positive roots of 3.4

$$
P_{s}=P_{s+}=-\mu+\sqrt{\mu^{2}+Q_{s}^{2}}
$$

we are led to a well-defined for $R>2 \mu$ solution with asymptotically flat metric and horizon at $R=2 \mu$. We note that $\left(A_{1}+A_{1}\right)$-dyon solution was considered earlier in [6, 8], see also [12, 19] for certain extensions.

\section{$3.2 \quad A_{2}$-case}

Let

$$
\lambda^{2}=3 / 2, \quad \varepsilon=1
$$

This value of dilatonic coupling constant appears after reduction to four dimensions of 5-dimensional Kaluza-Klein model. We get $h=1 / 2, a=-1$ and $(2.10)$ is the Cartan matrix for the Lie algebra $A_{2}=s l(3)$. In this case we obtain [15]

$$
H_{s}=1+\frac{P_{s}}{R}+\frac{P_{s}^{(2)}}{R^{2}}
$$


where

$$
\begin{array}{r}
2 Q_{s}^{2}=\frac{P_{s}\left(P_{s}+2 \mu\right)\left(P_{s}+4 \mu\right)}{P_{1}+P_{2}+4 \mu} \\
P_{s}^{(2)}=\frac{P_{s}\left(P_{s}+2 \mu\right) P_{\bar{s}}}{2\left(P_{1}+P_{2}+4 \mu\right)},
\end{array}
$$

$s=1,2(\bar{s}=2,1)$. The Kaluza-Klein uplift to $D=5$ gives us the well-known Gibbons-Wilthire solution [4], which is in an agreement with the general spherically-symmetric dyon solution (related to $A_{2}$ Toda chain) from [3].

\subsection{Special solution with equal charges}

There exists also a special solution

$$
H_{s}=\left(1+\frac{P}{R}\right)^{b},
$$

with equal charges $Q_{s}=Q, s=1,2$, satisfying

$$
Q^{2}=P(P+2 \mu)
$$

We remind that $b=h^{-1}$. For positive root of 3.11

$$
P=P_{+}=-\mu+\sqrt{\mu^{2}+Q^{2}},
$$

we get for $R>2 \mu$ a well-defined solution with asymptotically flat metric and horizon at $R=2 \mu$.

This solution is a special case of more general "block orthogonal" black brane solutions [26, 27]. Here the power in (3.10) appears due to relation

$$
b=2 \sum_{l=1,2} A^{s l}
$$

$s=1,2$, where $\left(A^{s l}\right)=\left(A_{s l}\right)^{-1}$. This power is integer for $A_{1}+A_{1}$ and $A_{2}$ cases.

It should be noted that this special solution is valid for both signes $\varepsilon= \pm 1$ and has a well-defined limit for $\lambda^{2}=1 / 2, \varepsilon=-1$ in agreement with Remark 1 (here $\left.\bar{H}_{s}=1+P / R\right)$. 


\subsection{The limiting $A_{1}$-case}

In what follows we will use two limiting solutions: electric one with $Q_{1}=$ $Q \neq 0$ and $Q_{2}=0$,

$$
H_{1}=1+\frac{P}{R}, \quad H_{2}=1,
$$

and magnetic one with $Q_{1}=0$ and $Q_{2}=Q \neq 0$,

$$
H_{1}=1, \quad H_{2}=1+\frac{P}{R} \text {. }
$$

In both cases $P=-\mu+\sqrt{\mu^{2}+b Q^{2}}$. These solutions correspond to the Lie algebra $A_{1}$. In various notations the solution (3.14) appeared earlier in [1] and [5, 6], and was extended to multidimensional case in [5, 6, 9, 10, ${ }^{1} \mathrm{~A}$ special case with $\lambda^{2}=1 / 2, \varepsilon=1$. was considered earlier in [2, 7].

\section{Physical parameters}

Here we consider certain physical parameters corresponding to the solutions under consideration

\subsection{Gravitational mass and scalar charge}

For (ADM) gravitational mass we get from 2.2

$$
G M=\mu+\frac{h}{2}\left(P_{1}+P_{2}\right),
$$

where parameters $P_{s}=P_{s}^{(1)}$ appear in the relation 3.1 and $G$ is the gravitational constant.

The scalar charge just follows from (2.3)

$$
Q_{\varphi}=\lambda h \varepsilon\left(P_{1}-P_{2}\right) .
$$

For the symmetric case $Q_{1}^{2}=Q_{2}^{2}=Q^{2}=P(P+2 \mu)$ with $P>0$ we get $P_{1}=P_{2}=b P$ and hence

\footnotetext{
${ }^{1}$ The results of [6] seems to be correct ones up to a typo in the first formula (2.1) for the action in 6] which should be eleminated: the kinetic term for the scalar field should be multiplied by extra factor $1 / 2$.
} 


$$
G M=\mu+P=\sqrt{\mu^{2}+Q^{2}}, \quad Q_{\varphi}=0 .
$$

In this case the gravitational mass and the scalar charge do not depend upon $\lambda$ and $\varepsilon$. The mass $M$ monotonically increases from $\mu$ (for $Q^{2}=+0$ ) to $+\infty$ (for $\left.Q^{2}=+\infty\right)$.

For fixed charges $Q_{s}$ and extremality parameter $\mu$ the mass $M$ and scalar charge $Q_{\varphi}$ are not independent but obey a certain constraint. Indeed, for fixed parameters $P_{s}=P_{s}^{(1)}$ in decomposition 3.1) we get

$$
y_{s}=\ln H_{s}=\frac{P_{s}}{2 \mu} z+O\left(z^{2}\right),
$$

for $z \rightarrow+0$, which after substitution into 2.14 gives us (for $z=0$ ) the following identity

$$
P_{1}^{2}+P_{2}^{2}+a P_{1} P_{2}+2 \mu\left(P_{1}+P_{2}\right)=b\left(Q_{1}^{2}+Q_{2}^{2}\right) .
$$

By using relations (4.1) and (4.2) this identity may be rewritten in the following form

$$
2(G M)^{2}+\varepsilon Q_{\varphi}^{2}=Q_{1}^{2}+Q_{2}^{2}+2 \mu^{2} .
$$

It is remarkable that this formula does not contain $\lambda$. We note that in the extremal case $\mu=+0$ this relation for $\varepsilon=1$ was obtained earlier in [11]. In derivation of (4.6) the following identities were used

$$
a+2=2 h, \quad 2-a=4 \varepsilon \lambda^{2} h .
$$

Remark 3. The paper [11] is an important one due to the following nontrivial result which was obtained numerically: for $\varepsilon=1$ the global extension of the metric 2.2. has two horizons only if $\lambda^{2}=p(p+1) / 4, p=1,2, \ldots$. Recently, this rule was explained in [23] in terms of analyticity of the dilaton at the $A d S^{2} \times S^{2}$ event horizon.

\subsection{The Hawking temperature and entropy}

The Hawking temperature corresponding to the solution is found to be

$$
T_{H}=\frac{1}{8 \pi \mu}\left(H_{10} H_{20}\right)^{-h},
$$


where $H_{s 0}$ are defined in 2.8. Here and in what follows we put $c=\hbar=\kappa=$ 1 .

For the symmetric case $Q_{1}^{2}=Q_{2}^{2}=Q^{2}=P(P+2 \mu)$ with $P>0$ we get

$$
T_{H}=\frac{1}{8 \pi \mu}\left(1+\frac{P}{2 \mu}\right)^{-2} \text {. }
$$

We see, that in this case the Hawking temperature $T_{H}$ does not depend upon the choice of $\lambda$ and $\varepsilon$. It monotonically decreases from $1 /(8 \pi \mu)$ (for $\left.Q^{2}=+0\right)$ to 0 (for $Q^{2}=+\infty$ ). (Here $\mu$ is fixed.)

The Bekenstein-Hawking (area) entropy $S=A /(4 G)$, corresponding to the horizon at $R=2 \mu$, where $A$ is the horizon area, reads

$$
S_{B H}=\frac{4 \pi \mu^{2}}{G}\left(H_{10} H_{20}\right)^{h} .
$$

It follows from 4.8 and 4.10 that the product

$$
T_{H} S_{B H}=\frac{\mu}{2 G}
$$

does not depend upon $\lambda, \varepsilon$ and charges $Q_{s}$. This product does not use explicit form of the moduli functions $H_{s}(R)$.

\subsection{PPN parameters}

Now we introduce a new radial variable $\rho$ by the relation $R=\rho(1+(\mu / 2 \rho))^{2}$ $(\rho>\mu / 2)$, which gives us the 3-dimensionally conformally-flat form of the metric 2.2

$$
g^{(4)}=U\left\{-U_{1} \frac{(1-(\mu / 2 \rho))^{2}}{(1+(\mu / 2 \rho))^{2}} d t \otimes d t+\left(1+\frac{\mu}{2 \rho}\right)^{4} \delta_{i j} d x^{i} \otimes d x^{j}\right\},
$$

where $\rho^{2}=|x|^{2}=\delta_{i j} x^{i} x^{j}(i, j=1,2,3)$ and

$$
U=\prod_{s=1,2} H_{s}^{h}, \quad U_{1}=\prod_{s=1,2} H_{s}^{-2 h} .
$$

The parametrized post-Newtonian (PPN) parameters $\beta$ and $\gamma$ are defined by following standard relations

$$
\begin{array}{r}
g_{00}^{(4)}=-\left(1-2 V+2 \beta V^{2}\right)+O\left(V^{3}\right), \\
g_{i j}^{(4)}=\delta_{i j}(1+2 \gamma V)+O\left(V^{2}\right),
\end{array}
$$


$i, j=1,2,3$, where, $V=G M / \rho$ is Newton's potential, $G$ is the gravitational constant and $M$ is the gravitational mass (in our case given by (4.1)).

The calculations of PPN (or Edington) parameters for the metric (4.12) give us the same result as in [18]:

$$
\beta=1+\frac{1}{4(G M)^{2}}\left(Q_{1}^{2}+Q_{2}^{2}\right), \quad \gamma=1 .
$$

These parameters do not depend upon $\lambda$ and $\varepsilon$. They may be calculated just without knowledge of explicit relations for functions $H_{s}(R)$.

It should be noted that (at least formally) these parameters obey the observational restrictions for the solar system [29], when the ratious $Q_{s} /(2 G M)$ are small enough.

\section{Bounds on mass and scalar charge, and nu- merical calculations}

Here we start with the following hypothesis which is supported by numerical calculations.

Conjecture. For any $h>0, \varepsilon= \pm 1, Q_{1} \neq 0, Q_{2} \neq 0$ and $\mu>$ 0: A) the moduli functions $H_{s}(R)$, which obey (2.7), (2.8) and (2.9), are uniquely defined and hence the parameters $P_{1}, P_{2}$, the gravitational mass $M$ and the scalar charge $Q_{\varphi}$ are uniquely defined too; $B$ ) the parameters $P_{1}, P_{2}$ are positive and the functions $P_{1}=P_{1}\left(Q_{1}^{2}, Q_{2}^{2}\right), P_{2}=P_{2}\left(Q_{1}^{2}, Q_{2}^{2}\right)$ define a diffeomorphism of $\left.\mathbb{R}_{+}^{2}\left(\mathbb{R}_{+}=\{x \mid x>0\}\right) ; C\right)$ in the limiting case we have:

(i) for $Q_{2}^{2} \rightarrow+0: P_{1} \rightarrow-\mu+\sqrt{\mu^{2}+b Q_{1}^{2}}, P_{2} \rightarrow+0$ and (ii) for $Q_{1}^{2} \rightarrow+0$ : $P_{1} \rightarrow+0, P_{2} \rightarrow-\mu+\sqrt{\mu^{2}+b Q_{2}^{2}}\left(b=h^{-1}\right)$.

The Conjecture could be readily verified for the case $\varepsilon=1, \lambda^{2}=1 / 2$. Another integrable case $\varepsilon=1, \lambda^{2}=3 / 2$ is more involved and it needs some efforts in verifying this conjecture.

It seems that the point B) is at the same time the most crucial and most difficult to prove. This is the main part of the conjecture.

For $h>0$ we are led to the following bounds on the gravitational mass $M$ and scalar charge $Q_{\varphi}\left(Q_{1} \neq 0, Q_{2} \neq 0\right)$

$$
\mu+\frac{h}{2}\left(-\mu+\sqrt{b\left(Q_{1}^{2}+Q_{2}^{2}\right)+\mu^{2}}\right)<G M \leq \sqrt{\frac{1}{2}\left(Q_{1}^{2}+Q_{2}^{2}\right)+\mu^{2}},
$$




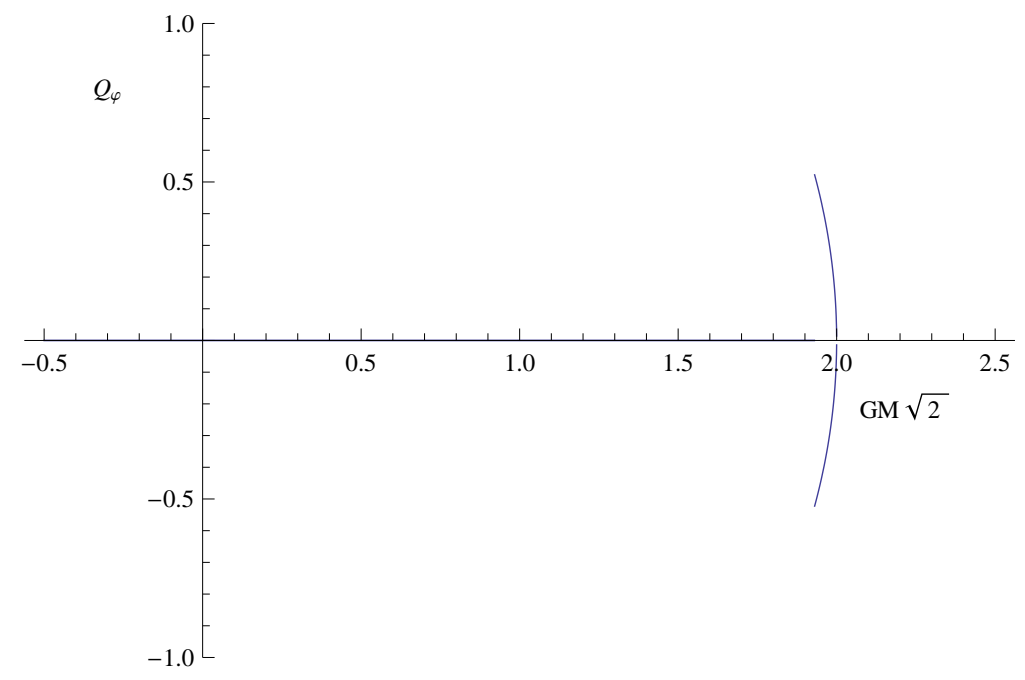

Figure 1: Graphical illustration of bounds on $M$ and $Q_{\varphi}$ for $\varepsilon=1, \lambda=1 / \sqrt{2}$, $\mu=1$ and $Q_{1}^{2}+Q_{2}^{2}=2$.

for $\varepsilon=+1(\lambda \neq 0,0<h<2)$,

$$
\sqrt{\frac{1}{2}\left(Q_{1}^{2}+Q_{2}^{2}\right)+\mu^{2}} \leq G M<\mu+\frac{h}{2}\left(-\mu+\sqrt{b\left(Q_{1}^{2}+Q_{2}^{2}\right)+\mu^{2}}\right),
$$

for $\varepsilon=-1\left(0<\lambda^{2}<\frac{1}{2}, h>2\right)$ and

$$
\left|Q_{\varphi}\right|<|\lambda| h\left(-\mu+\sqrt{b\left(Q_{1}^{2}+Q_{2}^{2}\right)+\mu^{2}}\right),
$$

which are valid for all $\lambda \neq 0$.

We illustrate the bounds on $M$ and $Q_{\varphi}$ graphically by two figures, which represent a set of physical parameters $G M$ and $Q_{\varphi}$ for $Q_{1}^{2}+Q_{2}^{2}=Q^{2}=2$ and $\mu=1$. Figure 1 corresponds to the case $\varepsilon=+1$ and $\lambda=\sqrt{\frac{1}{2}}$, while Figure 2 describes the limiting case $\varepsilon=-1$ and $\lambda=\sqrt{0.499}$. The middle points of these two arcs correspond to symmetric solutions with $Q_{1}^{2}=Q_{2}^{2}=1$, while the boundary points of the arcs correspond either to $Q_{1}^{2}=+0, Q_{2}^{2}=2$, or to $Q_{1}^{2}=2, Q_{2}^{2}=+0$.

Proof of the bounds. Let us prove the relations (5.1), (5.2) and (5.3) using the Conjecture. The right inequality (or equality) in (5.1) just follows 


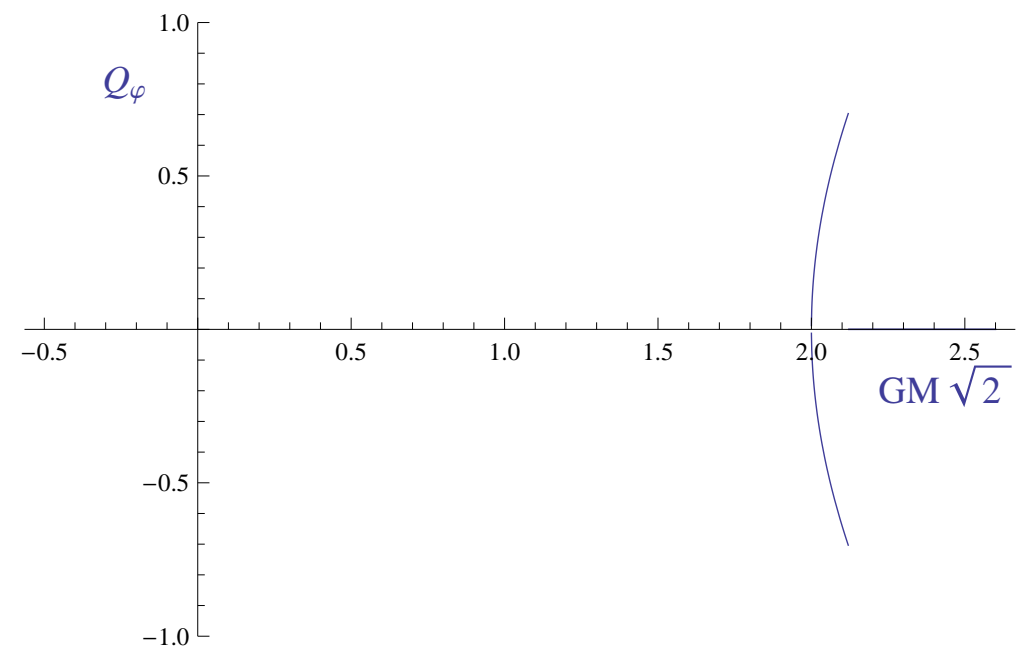

Figure 2: Graphical illustration of bounds on $M$ and $Q_{\varphi}$ for $\varepsilon=-1, \lambda=$ $\sqrt{0.499}, \mu=1$ and $Q_{1}^{2}+Q_{2}^{2}=2$.

from the relation (4.6), while the left inequality (or equality) in (5.2) follows from (4.6), and $M>0$ which is valid due to relation (4.1), $h>0$ and inequalities $P_{1}>0, P_{2}>0$ (due to Conjecture.). Now let us verify the left inequality in (5.1). We fix the charges by the relation $Q_{1}^{2}+Q_{2}^{2}=Q^{2}, Q>0$, and put $Q_{1}^{2}=\frac{1}{2} Q^{2}(1+x), Q_{2}^{2}=\frac{1}{2} Q^{2}(1-x)$, where $-1<x<1$. Due to 4.6 and $M>0$ we can use the following parametrization

$$
\sqrt{2} G M=R \cos \psi, \quad Q_{\varphi}=R \sin \psi, \quad R=\sqrt{Q^{2}+2 \mu^{2}},
$$

where $|\psi|<\pi / 2$. Owing to Conjecture and relations (4.1), (4.2) we get that $\psi=\psi(x)$ is a smooth function which obey

$$
\psi(0)=0, \quad \psi( \pm 1 \mp 0)= \pm \psi_{0} .
$$

Here $R \cos \psi_{0}=\sqrt{2}\left(\mu+\frac{h}{2} P\right)$ and $R \sin \psi_{0}=\lambda h P$, where $P=-\mu+$ $\sqrt{b Q^{2}+\mu^{2}}$. The limit $x \rightarrow+1-0$ corresponds to pure electric black hole while the limit $x \rightarrow-1+0$ corresponds to pure magnetic one. To prove the relations (5.1) and (5.3) one should verify the inequality

$$
-\psi_{0}<\psi(x)<\psi_{0}
$$

for all $x \in(-1,1)$. Let us suppose that (5.6) is not valid. Without loss of generality we put $\psi\left(x_{*}\right) \geq \psi_{0}$ for some $x_{*}$. Then using (5.5) and the 
smoothness of the function $\psi(x)$ we get that for some $x_{1} \neq x_{2}: \psi\left(x_{1}\right)=\psi\left(x_{2}\right)$. 2 Hence for two different sets $\left(Q_{1}^{2}, Q_{2}^{2}\right)_{1} \neq\left(Q_{1}^{2}, Q_{2}^{2}\right)_{2}$ we obtain the same coinciding sets: $\left(G M, Q_{\varphi}\right)_{1}=\left(G M, Q_{\varphi}\right)_{2}$ and $\left(P_{1}, P_{2}\right)_{1}=\left(P_{1}, P_{2}\right)_{2}$, see (4.1) and (4.2). But due to our Conjecture the map $\left(Q_{1}^{2}, Q_{2}^{2}\right) \mapsto\left(P_{1}, P_{2}\right)$ is bijective one. This implies $\left(P_{1}, P_{2}\right)_{1} \neq\left(P_{1}, P_{2}\right)_{2}$. We get a contradiction which proves our proposition for $\varepsilon=1$ and arbitrary $Q_{1}^{2}+Q_{2}^{2}>0$. The proof of right inequality in (5.2) and bounds (5.3) for $\varepsilon=-1$ is quite analogous one. The only difference here the use of parametrization

$$
\sqrt{2} G M=R \cosh \psi, \quad Q_{\varphi}=R \sinh \psi, \quad R=\sqrt{Q^{2}+2 \mu^{2}},
$$

instead of (5.4).

The inequalities (5.1), (5.2) and (5.3) imply the following bounds on mass and scalar charge, which are valid for all $\mu>0$ and $h>0$

$$
\frac{1}{2} \sqrt{h\left(Q_{1}^{2}+Q_{2}^{2}\right)}<G M
$$

for $\varepsilon=+1(\lambda \neq 0,0<h<2)$,

$$
\sqrt{\frac{1}{2}\left(Q_{1}^{2}+Q_{2}^{2}\right)}<G M
$$

for $\varepsilon=-1\left(0<\lambda^{2}<\frac{1}{2}, h>2\right)$, and

$$
\left|Q_{\varphi}\right|<|\lambda| \sqrt{h\left(Q_{1}^{2}+Q_{2}^{2}\right)}
$$

for both cases.

The bound (5.8) is in agreement with the bound (6.16) from ref. [30] (BPS-like inequality), which was proved there by using certain spinor techniques.

It should be noted that in the pure electric case for $\varepsilon=+1$ Gibbons and Wells have proved another bound which relates $M, Q_{\varphi},\left|Q_{1}\right|$ and $\lambda[31$. An open question is whether such bound could be extended somehow to the dyonic case.

\footnotetext{
${ }^{2}$ This may be readily proved by using Intermediate Value Theorem. This theorem states that if $f(x)$ is a continuous function on the interval $[a, b]$ then for any $d \in[f(a), f(b)]$, there is a point $c \in[a, b]$ such that $f(c)=d$. (Here for $f(a)>f(b),[f(a), f(b)]$ is meant as $[f(b), f(a)]$.)
} 
Remark 4. For $h<0$ the Conjecture is not valid. This may be verified just by analysing the solutions with small charge $Q_{1}$ (or $Q_{2}$ ).

Remark 5.It should be noted that here we are dealing with a very special class of solutions with phantom scalar field $(\varepsilon=-1)$. Even in the limiting case $Q_{2}=+0$ and $Q_{1} \neq 0$ there exist several branches of phantom black hole solutions which are not covered by our analysis [32] (see also [33].)

The bounds (5.1), (5.2) and (5.3) could be verified numerically by using the prescription which is described below. We outline some results of numerical calculations which are based on dynamical equations (2.11). We start with putting the boundary conditions on the horizon $z=1: y_{s}(1), s=1,2$.

Then for the first derivatives on the horizon $\left(\frac{d y_{s}}{d z}\right)_{\mid z=1}=y_{s}^{\prime}(1)$ we obtain from 2.11

$$
y_{s}^{\prime}(1)=b q_{s}^{2} \exp \left(-2 y_{s}(1)-a y_{\bar{s}}(1)\right),
$$

$s=1,2(\bar{s}=2,1)$. For practical calculations we put $z=1-\delta$, where $\delta$ is small enough, say $\delta=10^{-5}$, for initial values $y_{s}(1)$ about 1 . This is necessary for a correct formulation of the Cauchy problem for equations (2.11).

Our strategy is the following one. For fixed $\lambda$ and $\varepsilon$ we start with the exact symmetric solution obeing $y_{1}(0)=y_{2}(0)=0$, i.e. we put

$$
y_{1}(1)=y_{2}(1)=b \ln (1+p), \quad q_{1}^{2}=q_{2}^{2}=p(p+1) .
$$

See (3.10) and (3.11). Here $p=P /(2 \mu)>0$. Then we disturb relations (5.12) as follows

$$
y_{1}(1)=b \ln (1+p), \quad y_{2}(1)=k b \ln (1+p), \quad q_{1}^{2}=q_{2}^{2}=p(p+1),
$$

where $k \neq 1$. We get a numerical solution with $y_{1}(0)$ and $y_{2}(0)$ not obviously equal to 0.

Now, we make a shift in our solutions

$$
\bar{y}_{s}(z)=y_{s}(z)-y_{s}(0)
$$

$s=1,2$.

The functions $\bar{y}_{s}(z)$ give us a new solution to Toda-like equations (2.11) with rescaled charges

$$
\bar{q}_{s}^{2}=q_{s}^{2} \exp \left(2 y_{s}(0)+a y_{\bar{s}}(0)\right),
$$


$s=1,2$. The crucial point here is that $\bar{y}_{s}(z)$ obey the boundary conditions: $\bar{y}_{s}(0)=0, s=1,2$.

The asymptotical parameters $P_{s}$ are extracted from the relations (4.4) (with $y_{s}$ replaced by $\bar{y}_{s}$ ). The accuracy of calculations is controlled by (2.12) and 4.5).

Here we present certain examples of numerical data collected in Table 1 and Table 2. These data obey the bounds (5.1), (5.2) and (5.3). Of course, these tables may be enlarged by adding (a vast number of) new lines.

\begin{tabular}{|c|c|c|c|c|}
\hline$Q_{1}$ & $Q_{2}$ & GM & $Q_{\varphi}$ & bounds on $M$ and $Q_{\varphi}$ \\
\hline 0.233313 & 0.165107 & 1.03923 & 0.0128309 & true \\
0.233641 & 0.182372 & 1.0421 & 0.0100296 & true \\
0.234003 & 0.199861 & 1.04528 & 0.00693443 & true \\
0.234398 & 0.217596 & 1.04876 & 0.00353771 & true \\
0.234828 & 0.235605 & 1.05256 & -0.000169283 & true \\
0.235293 & 0.253911 & 1.05669 & -0.00419616 & true \\
\hline
\end{tabular}

Table 1: Examples of numerical calculations for $\varepsilon=1 ; \lambda=0.5 ; \mu=1$.

\begin{tabular}{|c|c|c|c|c|}
\hline$Q_{1}$ & $Q_{2}$ & GM & $Q_{\varphi}$ & bounds on $M$ and $Q_{\varphi}$ \\
\hline 0.00408717 & 0.0111095 & 1.00007 & 0.0000533559 & true \\
0.00408725 & 0.0122205 & 1.00008 & 0.0000663148 & true \\
0.00408733 & 0.0133316 & 1.0001 & 0.0000805081 & true \\
0.00408743 & 0.0144426 & 1.00011 & 0.0000959358 & true \\
0.00408753 & 0.0155537 & 1.00013 & 0.000112598 & true \\
0.00408764 & 0.0166649 & 1.00015 & 0.000130495 & true \\
\hline
\end{tabular}

Table 2: Examples of numerical calculations for $\varepsilon=-1 ; \lambda=0.5 ; \mu=1$.

\section{Conclusions}

In this paper a family of non-extremal black hole dyon solutions in a 4dimensional model with a scalar field is presented. The scalar field is either ordinary $(\varepsilon=+1)$ or ghost one $(\varepsilon=-1)$. The solutions are defined up to two functions $H_{1}(R)$ and $H_{2}(R)$, which obey two differential equations of second order with boundary conditions imposed. For $\varepsilon=+1$ these equations are 
integrable for two cases when $\lambda^{2}=1 / 2$ or $\lambda^{2}=3 / 2$. There is also a special solutions with coinciding electric and magnetic charges: $Q_{1}=Q_{2}$, which is defined for all (admissible) $\varepsilon$ and $\lambda$.

Here we have also calculated some physical parameters of the solutions: gravitational mass $M$, scalar charge $Q_{\varphi}$, Hawking temperature, black hole area entropy and post-Newtonian parameters $\beta, \gamma$. We have obtained a formula which relates $M, Q_{\varphi}$, dyon charges $Q_{1}, Q_{2}$, and the extremality parameter $\mu$ for all values of $\lambda \neq 0$. Remarkably, this formula does not contain $\lambda$. We have also shown that the product of the Hawking temperature and the Bekenstein-Hawking entropy does not depend upon $\varepsilon, \lambda$ and the moduli functions of the solutions $H_{s}(R)$ as well.

We have calculated the PPN parameters $\beta$ and $\gamma$ without knowledge of explicit formulas for $H_{s}(R)$. The only assumption was used that these functions are given (at least) by asymptotical series in $1 / R$ in the vicinity of the zero point. We have found that $\gamma=1$ and $\beta$ does not depend upon $\lambda$ and $\varepsilon$.

Here we have obtained bounds on gravitational mass and scalar charge for $1+2 \lambda^{2} \varepsilon>0$ which are based on the Conjecture (from Section 5) on parameters of solutions $P_{1}=P_{1}\left(Q_{1}^{2}, Q_{2}^{2}\right), P_{2}=P_{2}\left(Q_{1}^{2}, Q_{2}^{2}\right)$. We have also presented several results of numerical calculations which support our bounds. A rigorous proof of this conjecture may be a subject of a separate publication as well a detailed consideration of the case $\lambda^{2}>1 / 2, \varepsilon=-1$. For $\varepsilon=+1$ we have also deduced from our Conjecture the well-known (unsaturated) lower bound on mass, which was obtained earlier by Gibbons, Kastor, London, Townsend and Traschen [30] by using certain spinor techniques (just like in the well-known Nester-Witten approach).

An open question here is to find some physical (e.g. astrophysical) applications of the dyonic black hole solutions. Here one may consider a possible description of the black hole which is "located" at the Galactic Center. Recently, it was shown that near extremal Reissner-Nordström black hole provides a better fit of recent observational data for the black hole at the Galactic Center in comparison with the Schwarzschild black hole, see [34] and refs. therein. Dilatonic dyon black hole solutions (with certain scalar charge) may be used for a search of the best fit of the observational data for the black hole at the Galactic Center. For such research the thermodynamical calculations may be of relevance, e.g. due to possible analysis of the black hole stability, seach of bounds on variations of physical parameters etc. 
Acknowledgment The paper is supported by the program of KazNU (Almaty) on 2014 year. VD acknowledges support from a grant No. 0263/PCF 14 in fundamental research in natural sciences by the Ministry of Education and Science of Kazakhstan.

\section{References}

[1] K.A. Bronnikov and G.N. Shikin, On interacting fields in general relativity theory, Izvest. Vuzov (Fizika), 9, 25-30 (1977) [in Russian]; Russ. Phys. J. 20, 1138-1143 (1977).

[2] G.W. Gibbons, Antigravitating black hole solutions with scalar hair in $N=4$ supergravity, Nucl. Phys. B 207, 337-349 (1982).

[3] S.-C. Lee, Kaluza-Klein dyons and the Toda lattice, Phys. Lett. B 149, No 1-3, 98-99 (1984).

[4] G.W. Gibbons and D.L. Wiltshire, Spacetime as a membrane in higher dimensions, Nucl. Phys. B 287, 717-742 (1987).

[5] O. Heinrich, Charged black holes in compactified higher-dimensional EinsteinMaxwell theory, Astron. Nachr. 309, No 4, 249-251 (1988).

[6] G.W. Gibbons and K. Maeda, Black holes and membranes in higherdimensional theories with dilaton fields, Nucl. Phys. B 298, 741-775 (1988).

[7] D. Garfinkle, G. Horowitz and A. Strominger, Charged black holes in string theory, Phys. Rev. D 43, 3140 (1991); D45, 3888 (1992) (E).

[8] G.-J. Cheng, R.R. Hsu and W.-F. Lin, Dyonic Black Holes in String Theory, J. Math. Phys. 35, 4839-4847 (1994); hep-th/9302065.

[9] U. Bleyer, K.A. Bronnikov, S.B. Fadeev and V.N. Melnikov, Black hole stability in multidimensional gravity theory, Astron. Nachr. 315, No 4, 399-408 (1994); gr-qc/9405021.

[10] U. Bleyer and V.D. Ivashchuk, Mass bounds for Multidimensional Charged Dilatonic Black Holes, Phys. Lett. B 332, 292-296 (1994); gr-qc/9405018.

[11] S.J. Poletti, J. Twamley and D.L. Wiltshire, Charged Dilaton Black Holes with a Cosmological Constant, Phys. Rev. D 51, 5720-5724 (1995); hepth/9412076. 
[12] K.A.Bronnikov, On spherically symmetric solutions in D-dimensional dilaton gravity, Grav. Cosmol. 1, 67-78 (1995); gr-qc/9505020.

[13] H. Lü, C.N. Pope and K.W. Xu, Liouville and Toda solitons in M-Theory, Mod. Phys. Lett. A 11, 1785-1796 (1996); hep-th/9604058.

[14] V.D. Ivashchuk and V.N. Melnikov, P-brane black holes for general intersections, Grav. Cosmol. 5, No 4, 313-318 (1999); gr-qc/0002085.

[15] V.D. Ivashchuk and V.N. Melnikov, Black hole p-brane solutions for general intersection rules, Grav. Cosmol. 6, No 1, 27-40 (2000); hep-th/9910041.

[16] V.D. Ivashchuk and V.N. Melnikov, Toda p-brane black holes and polynomials related to Lie algebras, Class. Quantum Gravity 17, 2073-2092 (2000); math$\mathrm{ph} / 0002048$.

[17] V.D. Ivashchuk and V.N. Melnikov, Exact solutions in multidimensional gravity with antisymmetric forms, topical review, Class. Quantum Grav. 18, R1R66 (2001); hep-th/0110274.

[18] S.B. Fadeev, V.D. Ivashchuk, V.N. Melnikov and L.G. Sinanyan, On PPN parameters for dyonic black hole solutions, Grav. Cosmol. 7, No 4, 343-344 (2001).

[19] E. Kyriakopoulos, Black Holes in Models with Dilaton Field and Electric or Electric and Magnetic Charges, Class. Quant. Grav. 23, 7591-7602 (2006); gr-qc/0611061v1.

[20] H. Lü and W. Yang, SL(n,R)-Toda Black Holes, arxiv: 1307.2305.

[21] V.D. Ivashchuk, Black brane solutions governed by fluxbrane polynomials, Journal of Geometry and Physics, 86, 101-111 (2014); arxiv: 1401.0215.

[22] V.D. Ivashchuk and V.N. Melnikov, Multidimensional Gravity, Flux and Black Brane Solutions Governed by Polynomials, Grav. Cosmol. 20, No. 3, 182-189 (2014).

[23] D. Gal'tsov, M. Khramtsov and D. Orlov, "Triangular" extremal dilatonic dyons, Phys. Lett. B 743, 87-92 (2015); arXiv:1412.7709.

[24] N. Arkani-Hamed, H.-Ch. Cheng, M.A. Luty and S. Mukoyama, Ghost Condensation and a Consistent Infrared Modification of Gravity, JHEP 0405, 074 (2004); arxiv:hep-th/0312099. 
[25] E. Komatsu et al., Seven-Year Wilkinson Microwave Anisotropy Probe (WMAP) Observations: Cosmological Interpretation, Astrophys. J. Suppl. 192, 18 (2011); arxiv: 1001.4538[astro-ph].

[26] K.A. Bronnikov, Block-orthogonal Brane systems, Black Holes and Wormholes, Grav. Cosmol., 4, No 1, 49 (1998); hep-th/9710207.

[27] V.D. Ivashchuk and V.N. Melnikov, Multidimensional Cosmological and Spherically Symmetric Solutions with Intersecting $p$-branes, In: Lecture Notes in Physics, v.537. Mathematical and Quantum Aspects of Relativity and Cosmology.Eds.: S. Cotsakis and G. Gibbons. Springer, Berlin, 2000, p. 214; gr-qc/9901001.

[28] S. Cotsakis, V.D. Ivashchuk and V.N. Melnikov, P-branes Black Holes and Post-Newtonian Approximation, Grav. Cosmol. 5, No 1, (1999); grqc/9902148.

[29] C.M. Will, The Confrontation between General Relativity and Experiment, Living Rev. Relativity, 9, (2006), 3. [Online Article: cited 26.04.2015], http://www.livingreviews.org/lrr-2006-3.

[30] G.W. Gibbons, D. Kastor, L.A.J. London, P.K. Townsend and J. Traschen, Supersymmetric Self-Gravitating Solitons, Nucl. Phys. B 416, 850-880 (1994); arxiv: hep-th/9310118.

[31] G.W. Gibbons and C.G. Wells, Anti-Gravity Bounds and the Ricci Tensor, gr-qc/9310002.

[32] G. Clement, J.C. Fabris and M. Rodriges, Phantom black holes in EinsteinMaxwell-Dilaton theory, Phys. Rev. D 79, 064021 (2009); arxiv: 0901.4543.

[33] M. Azreg-Aïnou, G. Clément, J.C. Fabris and M.E. Rodrigues, Phantom Black Holes and Sigma Models, Phys. Rev. D 83: 124001, 12pp. (2011).

[34] A.F. Zakharov, Constraints on a charge in the Reissner-Nordström metric for the black hole at the Galactic Center, Phys. Rev. D 90: 062007, 8pp. (2014); arXiv:1407.7457. 